\title{
Anaesthesia in a patient with COVID-19 undergoing elective lower segment caesarean section: a case report
}

\author{
Keith SK Yeung *, MB, BS, CY Kwok, FHKCA, FHKAM (Anaesthesiology), YF Chow, FANZCA, FHKAM (Anaesthesiology)
}

This article was published on 11 Jun 2021 at www.hkmj.org.
Department of Anaesthesiology and Operating Theatre Services, Queen Elizabeth Hospital, Hong Kong

* Corresponding author: keithyeung31@gmail.com

\section{Case report}

In July 2020, a 35-year-old pregnant woman (weight $70 \mathrm{~kg}$, height $160 \mathrm{~cm}$, body mass index 27.3$)$ at $34+3$ weeks of gestation presented to our hospital with upper respiratory tract infection symptoms including dry cough and runny nose for 4 days. The patient had a history of lower segment caesarean section in 2016, with good past health and an unremarkable antenatal history. Reverse transcription polymerase chain reaction analysis of the patient's deep throat saliva sample was positive for severe acute respiratory syndrome coronavirus 2 (SARS-CoV-2). The cycle threshold value was 19.79 indicating a high viral load. She had no fever or shortness of breath at any time.

The patient also presented with a small amount of per-vaginal spotting but had no abdominal pain or leaking sensation. Fetal movements were active. The patient's blood pressure was $98 / 62 \mathrm{~mm} \mathrm{Hg}$, pulse was $97 \mathrm{bpm}, \mathrm{SpO}_{2}$ was $97 \%$ on room air, and body temperature was $37^{\circ} \mathrm{C}$. Fetal ultrasonography was unremarkable with appropriate size for gestational age. The impression was of antepartum haemorrhage of unknown origin and maternal coronavirus disease 2019 (COVID-19) infection.

The patient had normal liver and renal function, clotting profile and chest radiograph, and negative results for influenza $\mathrm{A} / \mathrm{B}$ and respiratory syncytial virus tests. Other results included: haemoglobin $11.1 \mathrm{~g} / \mathrm{dL}$, platelet count $198 \times 10^{9} / \mathrm{L}$, neutrophilia of $73 \%$ white blood cell differential count, lymphopenia at $0.9 \times 10^{9} / \mathrm{L} 16.5 \%$ of white blood cell differential count, and C-reactive protein $8 \mathrm{mg} / \mathrm{L}(\mathrm{ref}<5 \mathrm{mg} / \mathrm{L})$.

The patient was admitted to a negative pressure isolation ward, standard practice for airborne infection. A multidisciplinary meeting was held involving obstetricians, anaesthesiologists, paediatricians, microbiologists, infectious disease specialists, and intensivists. The consensus, agreed with the patient, was that an early elective lower segment caesarean section under spinal anaesthesia should be performed the next day.

Routes were isolated and protected for transferring patients between the operating theatre $(\mathrm{OT})$, the isolation ward, and the neonatal intensive care unit, and for transferring healthcare workers from the OT to decontamination shower facilities. Only essential equipment, medications, and consumables were placed inside the OT. Disposable consumables were used whenever possible. Paper records were minimised and sealed.

Preparations were made inside the OT for spinal anaesthesia, and for conversion to general anaesthesia if necessary. Medications for induction and resuscitation were prepared in advance, including propofol, succinylcholine, cisatracurium, phenylephrine, ephedrine, atropine, neostigmine, syntocinon, carbetocin, and tranexamic acid. A video laryngoscope with disposable blades, stylet, elastic gum bougie, endotracheal tubes and size 3 and 4 classic and supreme laryngeal masks were placed under plastic sheet covers.

Staff exposure time was minimised, but this was balanced against an optimal standard of patient care. Only active essential personnel were present in the OT for each stage of surgery. All staff inside the OT wore level 3 personal protective equipment, including fluid-resistant long-sleeve gown, gloves, face shield, and fit-tested N95 masks according to airborne precautions. During spinal anaesthesia, only two anaesthesiologists and an assistant were present in the OT. The anaesthesiologists wore water-resistant sterile gowns during the spinal anaesthesia. Other staff, including the surgeons and midwives, waited in the anteroom entering the OT only when the anaesthesia was complete.

The patient wore a water-resistant surgical mask throughout the transfer and surgery. She required no supplementary oxygen. On entering the OT, her blood pressure was $120 / 82 \mathrm{~mm} \mathrm{Hg}$, pulse $91 \mathrm{bpm}$, and $\mathrm{SpO}_{2} 99.4 \%$ on room air. Anaesthesia was induced by $2.2 \mathrm{~mL}$ of $0.5 \%$ hyperbaric bupivacaine, $15 \mu \mathrm{g}$ of fentanyl, and $0.15 \mathrm{mg}$ morphine injected intrathecally via a 25 -gauge pencil tip spinal needle in a single attempt, at the L3-L4 level, with sensory block to T5 bilaterally. Phenylephrine $(100 \mu \mathrm{g} / \mathrm{mL})$ infusion at $15 \mathrm{~mL} / \mathrm{h}(0.36 \mu \mathrm{g} / \mathrm{kg} / \mathrm{min})$ was commenced 5 minutes after spinal anaesthesia. A baby girl was delivered 19 minutes after the spinal injection. Total blood loss was $1000 \mathrm{~mL}$, and $2000 \mathrm{~mL}$ of Plasma- 
Lyte A was infused. A phenylephrine infusion was titrated down and stopped on completion of the surgery. The patient's blood pressure was 116/56 $\mathrm{mm} \mathrm{Hg}$ and her pulse was $68 \mathrm{bpm}$. Monitoring continued in the same OT to avoid contamination of the post-anaesthesia care unit.

Postoperative analgesia included oral paracetamol $1 \mathrm{~g}$ four times daily and diclofenac sodium sustained release $100 \mathrm{mg}$ daily. The patient was followed up daily. She was satisfied with the overall anaesthetic experience and pain control. The baby had 1-minute and 5-minute Apgar scores of 8. Repeated tests of nasopharyngeal aspiration and throat swab were negative for SARS-CoV-2 infection; however, the patient was nursed in an isolation ward in the neonatal intensive care unit as a precaution.

Samples including amniotic fluid, placental swab, high vaginal swab and breast milk all tested negative for SARS-CoV-2. All personnel directly involved in care of the mother and the baby and those involved in OT decontamination remained symptom free after 14 days of medical surveillance.

\section{Discussion}

The timing of delivery was a major concern for this patient. If the mother's health had deteriorated before delivery, the use of certain antiviral medications would have caused deranged organ function and affected fetal well-being. Corticosteroid may have harmed the mother. ${ }^{1,2}$ Use of corticosteroids in preparation for premature labour is thought to cause a worse outcome in patients with COVID-19. ${ }^{3}$ Fortunately our patient was at $>34$ weeks' gestation. Unexpected deterioration of the mother's health could also result in the need for an unanticipated urgent operative delivery, imposing increased operative risks, as well as increased infectious risks to healthcare workers. In particular, general anaesthesia requires endotracheal intubation that is considered an aerosol-generating procedure. Spinal anaesthesia offered a good alternative to general anaesthesia in a planned setting, as illustrated by a case series from Wuhan, China, of 49 caesarean deliveries with good blood pressure control achieved under spinal anaesthesia. ${ }^{4}$

Early data suggest that pregnant women do not develop more severe COVID-19. ${ }^{5}$ In a study of 241 births in the United States, approximately 30\% of mothers with COVID-19 became symptomatic, $7.1 \%$ required intensive care unit admission, 3.7\% required intubation, and $0.7 \%$ progressed to a critical condition. The deterioration could be rapid and occurred over a variable time frame. ${ }^{5}$ Current guidelines suggest that COVID-19 infection by itself is not an indication for early induction of labour or operative delivery, but that the timing of delivery should be determined by obstetric indications. ${ }^{6}$

This patient presented with antepartum haemorrhage of unknown origin, and minor placental abruption could not be excluded. Any deterioration in placental abruption could rapidly jeopardise the well-being of the fetus and mother. The decision for early elective operative delivery was appropriate in these circumstances.

Concern was expressed about high viral load and infectivity, given the patient's high cycle threshold value of 19.79. However, there is wide variation in the interpretation of cycle threshold, ${ }^{7}$ and no evidence that a lower cycle threshold value correlates with worse prognosis. ${ }^{8}$

This case report confirms that it is possible for a patient with confirmed SARS-CoV-2 infection to safely undergo spinal anaesthesia with maintenance of stable blood pressure. ${ }^{4}$ Our findings suggest that pregnant women with mild COVID-19 symptoms are little different to healthy pregnant women who undergo spinal anaesthesia for caesarean section.

Overall, this case report demonstrates that with the input of anaesthesiologists, joint clinical decisions and effective communication between relevant specialties, a well-planned and rehearsed routing, and correct use of personal protective equipment, the infectious risks to health care professionals could be minimised while providing an appropriate standard of care to the mother and the baby.

\section{Author contributions}

All authors contributed to the concept or design of the study, acquisition of the data, analysis or interpretation of the data, drafting of the manuscript, and critical revision of the manuscript for important intellectual content. All authors had full access to the data, contributed to the study, approved the final version for publication, and take responsibility for its accuracy and integrity.

\section{Conflicts of interest}

The authors declare that they have no conflict of interest.

\section{Funding/support}

This case report received no specific grant from any funding agency in the public, commercial, or not-for-profit sectors.

\section{Ethics approval}

The patient was treated in accordance with the tenets of the Declaration of Helsinki. The patient provided written informed consent for all treatments and procedures.

\section{References}

1. Sanders JM, Monogue ML, Jodlowski TZ, Cutrell JB. Pharmacologic treatments for coronavirus disease 2019 (COVID-19): a review. JAMA 2020;323:1824-36.

2. Bauer ME, Bernstein $\mathrm{K}$, Dinges $\mathrm{E}$, et al. Obstetric anesthesia during the COVID-19 pandemic. Anesth Analg 2020;131:7-15.

3. Society for Maternal-Fetal Medicine, Society for Obstetric and Anesthesia and Perinatology. Labor and delivery 
COVID-19 considerations. Available from: https:// s3.amazonaws.com/cdn.smfm.org/media/2402/SMFMSOAP_COVID_LD_Considerations_-_revision_6-16-20_ PDF.pdf. Accessed 26 Jul 2020.

4. Zhong Q, Liu YY, Luo Q, et al. Spinal anaesthesia for patients with coronavirus disease 2019 and possible transmission rates in anaesthetists: retrospective, single-centre, observational cohort study. Br J Anaesth 2020;124:670-5.

5. Khoury R, Bernstein PS, Debolt C, et al. Characteristics and outcomes of 241 births to women with severe acute respiratory syndrome coronavirus 2 (SARS-CoV-2) infection at five New York City Medical Centers. Obstet Gynecol 2020;136:273-82.

6. National Institutes of Health. Coronavirus disease 2019 (COVID-19) treatment guidelines. Available from: https:// www.covid19treatmentguidelines.nih.gov/. Accessed 25 Jul 2020.

7. Han MS, Byun JH, Cho Y, Rim JH. RT-PCR for SARS-CoV-2: quantitative versus qualitative. Lancet Infect Dis 2021;21:165.

8. Young BE, Ong SW, Kalimuddin S, et al. Epidemiologic features and clinical course of patients infected with SARS-CoV-2 in Singapore. JAMA 2020;323:1488-94.

\section{Answers to CME Programme Hong Kong Medical Journal April 2021 issue}

Hong Kong Med J 2021;27:99-105

I. Findings from the first public COVID-19 temporary test centre in Hong Kong
A
1. False
2. False
3. True
4. True
5. False
B
1. False
2. True
3. False
4. False
5. True

Hong Kong Med J 2021;27:127-39

II. Hong Kong Geriatrics Society and Hong Kong Urological Association consensus on personalised management of male lower urinary tract symptoms in the era of multiple co-morbidities and polypharmacy
A
1. True
2. True
3. True
4. True
5. False
B
1. False
2. True
3. False
4. True
5. False 